\title{
Análisis de las horas de sueño y su repercusión en el desempeño académico de jóvenes de Bachillerato
}

\section{Analysis of sleep hours and their impact on the academic performance of high school students}

\author{
SESENTO, Leticia* $\dagger^{1} \&$ LUCIO, Rodolfo ${ }^{2}$ \\ ${ }^{1}$ Colegio Primitivo y Nacional de San Nicolás de Hidalgo \\ ${ }^{2}$ Facultad de Medicina Veterinaria y Zootecnia de la Universidad Michoacana de San Nicolás de Hidalgo \\ ID $1^{\text {er }}$ Autor: Leticia, Sesento / ORC ID: 0000-0002-6456058X, Researcher ID Thomson: S-6997-2018, CVU CONACYT \\ ID: 449302 \\ ID $1^{\mathrm{er}}$ Autor: Rodolfo, Lucio / ORC ID: 0000-0002-0535-3652, Researcher ID Thomson: X-2391-2018
}

\section{Resumen}

El sueño es parte vital del desarrollo biológico de todos los seres vivos, si bien, con el ritmo actual de los jóvenes, las redes sociales, series televisivas, fiestas, etc, cada día disminuyen las horas de sueño. El objetivo de este estudio es conocer los trastornos de sueño y su impacto en el rendimiento académico en estudiantes de bachillerato; los sujetos encuestados fueron 112. La metodología cuantitativa, de corte descriptivo. La hora promedio en la que suelen dormir los jóvenes son 12 pm (58 encuestados), 28 jóvenes duermen 8 horas, 69 duerme 6 horas y 15 de ellos sólo 4 horas al día. La mayoría de los jóvenes (63) tiene un promedio regular de 7 a 8.9, lo cual, puede tener diversos factores, pero asociando las encuestas los que padecen algún tipo de trastorno o pocas horas de sueño afirman tener promedio de malo a regular. Es importante realizar programas de intervención en el aula y en la Institución para concientizar sobre la importancia del sueño tanto a nivel salud como a nivel académico.

\section{Sueño, Desempeño, Tutoría}

\begin{abstract}
The dream is a vital part of the biological development of all living beings, although, with the current rhythm of young people, social networks, television series, parties, etc., daily sleep hours decrease. The objective of this study is to know sleep disorders and their impact on academic performance in high school students; the subjects surveyed were 112. The quantitative methodology, descriptive cut. The average time that young people usually sleep is $12 \mathrm{pm}$ (58 respondents), 28 young people sleep 8 hours, 69 sleep 6 hours and 15 of them only 4 hours a day. The majority of young people (63) have a regular average of 7 to 8.9 , which may have several factors, but associating the surveys those who suffer some type of disorder or a few hours of sleep claim to have average of bad to regular. It is important to carry out intervention programs in the classroom and at the Institution to raise awareness about the importance of sleep both at the health level and at the academic level.
\end{abstract}

Dream, Performance, Mentoring

Citación: SESENTO, Leticia \& LUCIO, Rodolfo. Análisis de las horas de sueño y su repercusión en el desempeño académico de jóvenes de Bachillerato. Revista de Pedagogía Crítica. 2019, 3-9: 1-8

\footnotetext{
* Correspondencia al Autor (Correo electrónico: leticiasesentogarcia@yahoo.com.mx)

$\dagger$ Investigador contribuyendo como primer autor.
} 


\section{Introducción}

La adolescencia es un período de importantes cambios biológicos hormonales y de crecimiento; acompañado de un amplio rango de cambios emocionales, psicológicos y sociales, que pueden producir en algunos adolescentes una serie de trastornos adaptativos, esta es la época inicial de la maduración sexual, el paso de niño a adulto (la adolescencia). El sueño es la ruptura fisiológica del estado de conciencia y uno de los cambios básicos que ocurren en el sistema nervioso. Es esencial para mantener un equilibrio de la psicología humana, por lo que su carencia provoca daños significativos en la concentración, en la memoria y en el control emocional.

Hoy en día la falta de sueño entre adolescentes es frecuente y se relaciona con problemas escolares o problemas de en la vida, la mayoría de los adolescentes no duermen lo suficiente, habitualmente porque tienen horarios sobrecargados o porque pasan demasiado tiempo enviando mensajes de texto o chateando con amigos hasta la madrugada. Otros adolescentes intentan acostarse temprano pero, en lugar de conciliar el sueño y dormir las horas que necesitan, se pasan horas acostados pero despiertos.

La reducción del sueño por malos hábitos o por trastornos del sueño conduce 6 con frecuencia el cansancio físico y esto repercute en la calidad de vida, menos rendimiento académico. "La primer clasificación de trastornos del sueño se publicó en 1979, al cabo de todos estos años actualmente se usa la clasificación internacional de los trastornos del sueño publicada en 2014”. (Correa, 2014)

\section{Justificación}

El sueño es imprescindible para lograr y mantener un correcto rendimiento académico, mental y físico del ser humano, pues este ayuda a fortalecer en la memoria y aprendizaje. Es posible que muchos de los jóvenes asistan a clases sin haber dormido suficiente la noche anterior, las causas podrían ser variadas desde hábitos en casa, preocupación por actividades académicas y situaciones personales, hasta problemas familiares o de salud.
Conocer algunas de las principales razones por las que los alumnos de bachillerato duermen poco o su sueño es ineficiente, podría darnos indicadores de cómo abordar la situación con jóvenes con bajo rendimiento escolar y estos sean referidos con profesionales que puedan dar solución a la problemática, evitar que los jóvenes tomen la decisión de auto medicarse o usar remedios o sustancias que les ayuden a conciliar el sueño.

\section{Problema}

Las escuelas preparatorias pretenden formar jóvenes capacitados con un rendimiento académico favorable, sin embargo existen diversos aspectos que influyen de manera desfavorable durante la formación y la preparación del estudiante. El sueño es un fenómeno elemental de la vida y una fase indispensable de la existencia humana. Es la necesidad fisiológica vital para el correcto funcionamiento del organismo o uno de los cambios o fenómenos básicos que ocurren en el sistema nervioso en cada ciclo de 24 horas. Hoy día, la falta de sueño entre los adolescentes es frecuente y se relaciona con problemas escolares o hábitos de vida. Por esa razón hemos decidido hablar de este tema ya que queremos lograr que haya conciencia que un buen descanso aportara un buen rendimiento académico de los jóvenes y buena salud.

Tener un descanso inadecuado tiene efectos en las personas y los principales problemas en los que se enfoca el presente son: la somnolencia y la fatiga, los cuales repercuten en el aprendizaje. Otros efectos que se busca destacar que provocan la pérdida del sueño son: cambios de humor, depresión, dificultad para concentrarse, estrés, ansiedad y efectos negativos sobre la salud física.

\section{Hipótesis y variables}

Hipótesis: Los jóvenes del nivel bachillerato presentan insomnio como trastorno de sueño debido al uso excesivo de redes sociales y repercute en un bajo rendimiento académico.

Variable independiente: Redes sociales.Son plataformas web abiertas que permiten la interacción entre muchos usuarios a partir de la creación de perfiles e intercambio de mensajes, videos o imágenes. 
Variables dependientes: Insomnio.Trastorno de sueño donde las personas sufren de no conciliar el sueño.

Rendimiento académico.- Es la evaluación del conocimiento adquirido en el ámbito escolar.

\section{Objetivo}

Conocer los trastornos del sueño que presentan los alumnos de bachillerato turno matutino y sus repercusiones en el rendimiento académico.

\section{Marco teórico}

\section{El Sueño}

El sueño es un fenómeno elemental de la vida y una fase indispensable de la existencia humana. Todos los individuos requieren de un periodo de descanso para afrontar las actividades que se presentan diariamente. En los estudiantes, es esencial para recuperar las fuerzas necesarias para rendir eficazmente en situaciones escolares, sociales o personales.

Cualquier situación relacionada con la vida diaria, ya sea en el ámbito académico, personal o laboral, será un factor que predisponga a la persona a padecer algún trastorno o problema del sueño. Un trastorno se define como una variedad de afecciones que influyen en el estado de ánimo, el pensamiento y el comportamiento, que perjudicará también de forma significativa en el desarrollo saludable del individuo, se altera el funcionamiento regular en el organismo a nivel psíquico y mental.

La influencia paternal es fundamental en el crecimiento y desarrollo de los adolescentes, sin embargo existen estudios que determinan que sólo un 5\% de los adolescentes siguen una rutina de sueño pautada por sus padres, siendo lo más habitual que se acuesten cuando hayan terminado sus deberes, su cena, sus relaciones sociales o sencillamente cuando ellos mismos sienten que tienen sueño. Esto hace que aparezca una cierta anarquía de horarios y un sueño frecuentemente insuficiente (García, 2004) En la adolescencia pareciera que los jóvenes requieren menos horas de sueño, pero en realidad son necesarias alrededor de 10 horas, no descansar lo suficiente podría provocar que el adolescente padezca una serie de problemas que afectarían en los diferentes ambientes en los que el individuo se desenvuelve.
Los adolescentes precisan dormir $9-10$ horas de sueño nocturno todos los días de la semana para rendir adecuadamente durante el día. "Fisiológicamente, el sueño del adolescente presenta un menor porcentaje de sueño lento profundo que desciende hasta en un $40 \%$ desde la edad prepuberal a la edad adulta, tiempo en el que se termina de desarrollar el cuerpo humano que oscila de entre los 10 a los 19 años de edad". (Mental,2002)

\section{Funciones del sueño}

Una de las funciones más importantes del sueño es su contribución en la regulación de la temperatura corporal, funcionando como un termostato que mantiene la temperatura que el organismo necesita en cada momento en función de las actividades que se llevan a cabo en él para facilitar procesos metabólicos, hormonales, etc. Sin este importante termostato, el organismo moriría.

El sueño produce dos tipos principales de acciones fisiológicas: en primer lugar, efectos sobre el propio sistema nervioso, en segundo lugar, efectos sobre otros sistemas funcionales del cuerpo. La vigilia prolongada suele asociarse a una disfunción progresiva de los procesos mentales $y$ en ocasiones da lugar a comportamientos anormales. Podríamos proponer que el valor principal del sueño consiste en reestablecer los equilibrios naturales entre centros neuronales.

Entre las funciones más destacadas del sueño podemos destacar las siguientes: Reestablecer almacenes de energía celular, restaurar la enostasis del Sistema Nervioso Central y del resto de tejidos. De modo resumido podríamos decir que dormimos para poder estar despiertos por el día y que, precisamente porque estamos despiertos $\mathrm{y}$ activos durante el día necesitamos dormir. El sueño es una necesidad básica del organismo y su satisfacción nos permite la supervivencia.

Todo lo que pasa en el cuerpo humano guarda un equilibrio, y si falla este equilibrio el organismo tratará por todos los medios de volver a recuperarlo. Gracias a los experimentos de privación de sueño se ha comprendido que cuando se elimina "completamente" la posibilidad de dormir en un organismo, sobreviene la muerte (García, 2004) 


\section{Trastornos del sueño}

Es todo problema de sueño, todo cambio o alteración en los hábitos, que afectan el desarrollo habitual del ciclo sueño-vigilia. Estos incluyen dificultades para conciliar el sueño o permanecer dormido, quedarse dormido en momentos inapropiados, dormir demasiado y conductas anormales del sueño.

Los trastornos del sueño son aquellos que afectan la capacidad para dormirse o para mantenerse dormido, y provocan que se duerma demasiado o se produzcan conductas anormales asociadas al sueño, son comunes en adolescentes y pueden ser temporales, intermitentes o crónicos. "Se puede entender como la disolución fisiológica del estado de conciencia o como uno de los cambios o fenómenos básicos que ocurren en el sistema nervioso en cada ciclo de 24 horas, se ha encontrado una prevalencia de 0,2 a $10 \%$ en el sexo femenino y la progresión en edad también es un factor de riesgo" (Mental,2002).

Los síntomas asociados a trastornos del sueño incluyen dificultad para mantener el sueño, presentar sueño o llegar a dormir en situaciones no propicias para el mismo, experimentar excesivo sueño durante los periodos de vigilia o cualquier manifestación de conductas anormales durante el mismo. Estos trastornos se acompañan con frecuencia de depresión, ansiedad y cambios cognitivos que se deben tener en cuenta en la planificación del tratamiento y en su manejo. Las alteraciones persistentes del sueño son factores de riesgo establecidos para el desarrollo posterior de enfermedades mentales y trastornos por consumo de sustancias.

\section{Areas del cerebro involucradas en el sueño}

La parte importante del cerebro involucrada en la coordinación del proceso del sueño se denomina Sistema Activador Reticular comprende un gran número de células nerviosas del cerebro y es el centro del Sistema Nervioso Central, responsable de regular la vigilia y las funciones vitales durante el sueño. Cuando las neuronas "apagan" su actividad, el Sistema Activador Reticular reduce el nivel de lucidez mental. Hay una interacción continua y significativa entre el Sistema Activador Reticular y otros órganos reguladores del cerebro.
Estos incluyen el tálamo, la médula espinal, la glándula pineal, los núcleos del rafe, el prosencéfalo basal, el hipocampo y el núcleo supraquiasmático.

Las áreas del cerebro que se encuentran directamente relacionadas con el sueño fueron descubiertas al comienzo del siglo XX (Barón Constantin):

Etapa de sueño: encontrándose implicada la región anterior al hipotálamo.

Etapa de vigilia formadas por la región posterior de hipotálamo.

Etapa de vigilia-sueño constituido por dos fases: la formación reticular ascendente y los núcleos del hipotálamo posterior central.

Desactivación del estado de vigilia: Medula oblonga Núcleos pre óptico del hipotálamo.

\section{Sonambulismo}

Es un trastorno del sueño que se origina durante el sueño profundo y resulta en caminar u otros comportamientos complejos. Es más común en niños y adolescentes que en adultos y es más probable que ocurra si una persona no ha dormido lo suficiente debido a que un sonámbulo típicamente permanece en un sueño profundo durante el episodio, puede ser difícil despertarlo y probablemente no va a recordar el incidente sonambulistico. La característica esencial del sonambulismo es la presencia de episodios repetidos de conducta motora compleja que se inician durante el sueño, entre las que están en levantarse de la cama y caminar (Márquez,2013)

\section{Trastorno de insomnio}

El insomnio se define como la dificultad para conciliar el sueño o alteraciones en el patrón de sueño que llevan a la percepción de que éste es insuficiente en su duración, profundidad o propiedades reparadoras. Éste puede ser inicial dificultad para dormirse y se asocia con frecuencia a trastornos emocionales como la ansiedad, la fobia y la depresión. Puede ser al inicio de la noche (cuando no te puedes dormir) o bien con despertarse a lo largo de la noche. 
La principal causa en la adolescencia es una higiene de sueño inadecuada. ya que en la adolescencia el consumo de tabaco, cafeína o alcohol son factores importantes y negativos sobre el mantenimiento del sueño. Hoy que saber que la desorganización y el estrés familiar y un patrón de sueño alterado en los padres afecta negativamente al sueño del adolescente. Si el adolescente no duerme bien esta irritable, le cuesta despertarse, recupera el sueño durante el fin de semana. Todo esto tiene efectos negativos sobre su vida. Por ello es importante detectarlo y actuar (Márquez, 2013)

\section{Apnea obstructiva del sueño}

La apnea obstructiva del sueño es un trastorno del sueño en que la respiración se detiene repetidas veces porque las vías respiratorias se bloquean. Consiste en la interrupción, parcial o completa de la respiración durante más de 10 segundos y se manifiesta durante el sueño, y es debido a que se produce estrechamiento en la vía aérea superior (garganta) este tipo de apnea es la más frecuente y es una enfermedad crónica. Los síntomas comunes incluyen ronquidos y un resoplido o jadeo estridente para respirar tras una pausa en la respiración, lo que puede ocurrir hasta 30 veces en una hora (Alva,2009)

\section{Síndrome de piernas inquietas}

El Síndrome de Piernas Inquietas lo ubicamos dentro de los trastornos del movimiento y se caracteriza por una urgencia para mover las piernas debida a una sensación de incomodidad (presión, cosquilleo etc.), que aparece en situaciones de reposo y que mejora claramente con la deambulación o el movimiento de las piernas estos suelen ser intensos de madrugada y se asocian a una sensación desagradable. Estos síntomas aparecen o empeoran al final del día. Se alivian con el movimiento y tiene gran impacto en la calidad de vida, les causa insomnio, fatiga diurna, disminución de la atención e hiperactiva paradójica y, en casos graves, somnolencia diurna excesiva. Tiene un predominio vespertino, por lo que la mayor intensidad de los síntomas se percibe a última hora del día o ya en la cama, el Síndrome de Piernas Inquietas es más habitual en personas de edad avanzada pero se ha asociado a insomnio y más recientemente a déficit de atención e hiperactividad en niños y adolescentes La causa no son del todo conocidas. Los adolescentes que padecen este síndrome pueden tener niveles bajos de hierro en la sangre.

ISSN 2523-2479

ECORFAN $^{\circledR}$ Todos los derechos reservados
La base del tratamiento es tener buenos hábitos del sueño. En este caso de que sea necesario, un especialista en trastornos del sueño podrá indicar algún tratamiento farmacológico ( P. Rubí, 2018)

\section{Metodología de la investigación}

\section{Tipo de investigación}

En la investigación realizada se utilizara el método cuantitativo. Un método cuantitativo es un procedimiento que se basa en la utilización de los números para analizar, investigar y comprobar tanto información como dados.

La investigación cuantitativa tiene como finalidad obtener respuestas de los estudiantes a preguntas específicas.

Características del método cuantitativo:

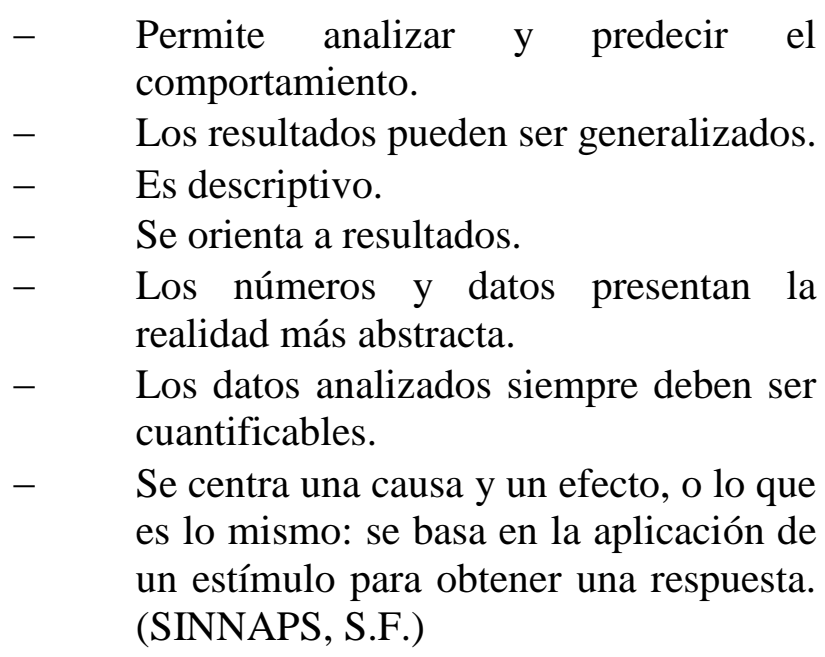

\section{Muestra}

Se encuestó a 112 jóvenes de bachillerato turno matutino, jóvenes de ambos sexos y diversos semestres $\left(2^{\circ}, 4^{\circ}\right.$ y $\left.6^{\circ}\right)$.

\section{Instrumento}

El instrumento empleado fue diseñado por los tutores pares en turno, y se integra de 15 reactivos, para determinar si presentan trastornos del sueño y si se percatan de la relación entre sueño y desempeño académico.

1. ¿Hora en la que duerme habitualmente?

a) 10:00 a.m. b) 12:00 a.m. c) 2:00 a.m.

2. ¿Tiene dificultad para conciliar el sueño?

a) Si b) No

SESENTO, Leticia \& LUCIO, Rodolfo. Análisis de las horas de sueño y su repercusión en el desempeño académico de jóvenes de Bachillerato. Revista de Pedagogía Crítica. 2019 
3. ¿Cuántas horas duerme normalmente?

a) 8 horas b) 6 horas c) 4 horas

4. ¿Tiene pesadillas constantemente?

a) Si b) No

5. ¿Durante el último mes cuantas veces has tenido problemas para dormir?

a) 5 b) 10 c) 15

6. ¿Suele despertarse durante la noche?

a) Ninguna en el último mes b) Una o dos veces por semana c) Tres o más veces por semana

7. ¿Ronca mientras duerme?

a) Ninguna el último mes b) Una o dos veces por semana c) Tres o más veces por semana

8. ¿Alguna vez ha tomado medicamento para dormir?

a) $\mathrm{Si}$ b) No

9. ¿Cuántas veces por semana consume medicamento para conciliar el sueño?

a) Todos los días b) Nunca c) Otra

10. ¿Haces siesta por las tardes?

a) $\mathrm{Si}$ b) No

11. ¿Cuál es tu promedio escolar actual?

a) 6 a 7.9 b) 7 a 8.9 c) 9 a 10

12. ¿Cómo consideras tu rendimiento escolar?

a) Bueno b) Regular c) Malo

13. ¿Consideras que las horas que descansas influyen en tu rendimiento académico?

a) $\mathrm{Si}$ b) $\mathrm{No}$

14. ¿Tienes problemas para mantener la atención en las actividades escolares?

a) $\mathrm{Si}$ b) $\mathrm{No}$

15. ¿Tienes problemas para mantenerte alerta en horario de clases?

a) $\mathrm{Si}$ b) $\mathrm{No}$

\section{Resultados}

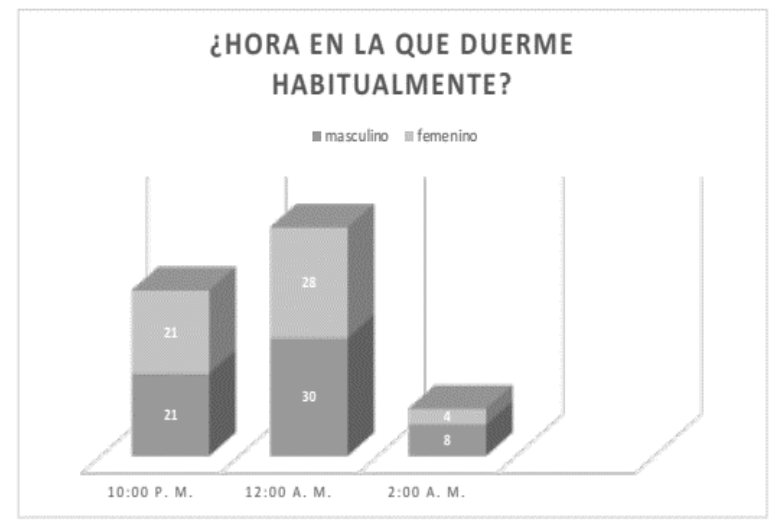

Grafico 1

En la pregunta número uno, se tiene el objetivo de analizar los hábitos de sueño de los adolescentes estudiantes de bachillerato, con esto se busca corroborar certeramente si la calidad del sueño influye en el rendimiento escolar.

En relación a la pregunta 1 que cuestiona a los 112 encuestados sobre la hora en la que duerme habitualmente, nos da como resultado que, 21 alumnos duermen a las 10:00 P. M. 21 alumnas duermen a las 10:00 P. M. Además, 30 alumnos duermen a las 12:00 A. M. y 28 alumnas también lo hacen. Por ultimo 8 alumnos duermen a las 2:00 A. M. y 4 alumnas duermen a las 2:00 A. M. En los resultados arrojados en esta pregunta, se observa que son los varones quienes tienen la tendencia a dormir más tarde.

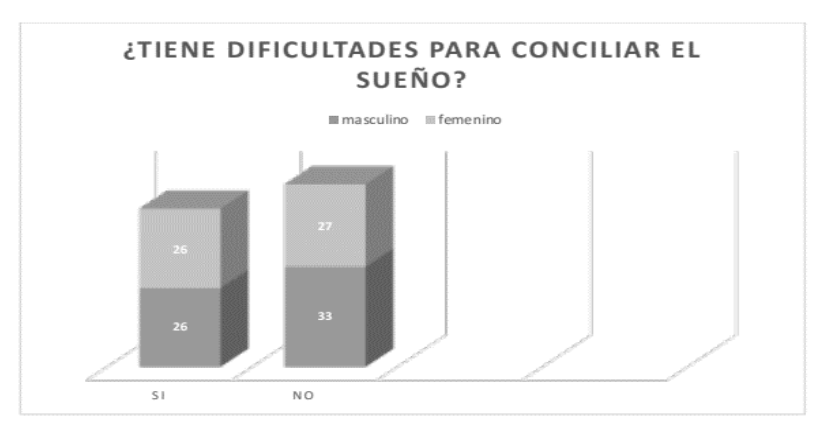

Grafico 2

Lo anterior muestra que 52 alumnos si presentan dificultades para conciliar el sueño, y por otro lado, 60 consideran no tener ninguna dificultad. 


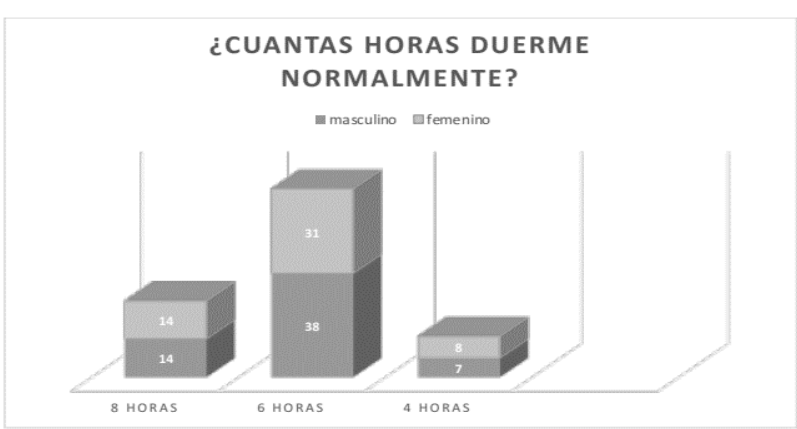

Grafico 3

Aquí se observa que, 28 jóvenes duermen las 8 horas recomendadas, 69 de ellos duerme 6 horas y 15 de ellos tan sólo duermen 4 horas al día. Los resultados obtenidos muestran que gran parte de los encuestados tienen hábitos de sueño que son insuficientes según lo recomendado, al no cumplir con las ocho horas que son medicamente suficientes para que el cuerpo logre un descanso óptimo.

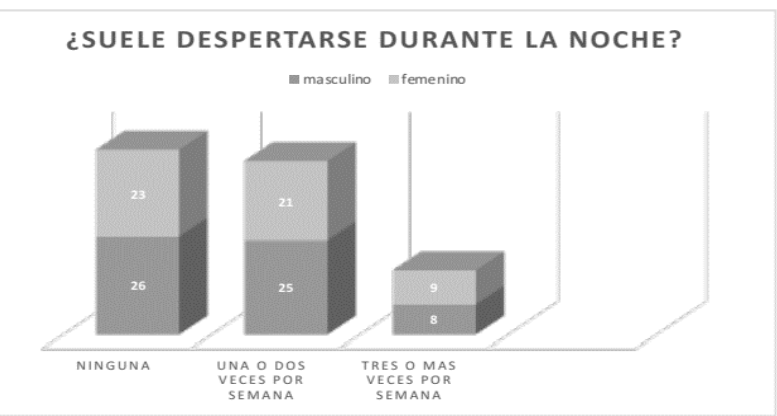

Grafico 4

En la anterior grafica se muestra que, 49 jóvenes no suelen despertarse durante la noche, mientras que 46 afirman que lo hacen una o dos veces por semana y 17 de ellos suele despertarse tres o más veces por semana. Es notable que esta dificultad del sueño es común entre los estudiantes de bachillerato.

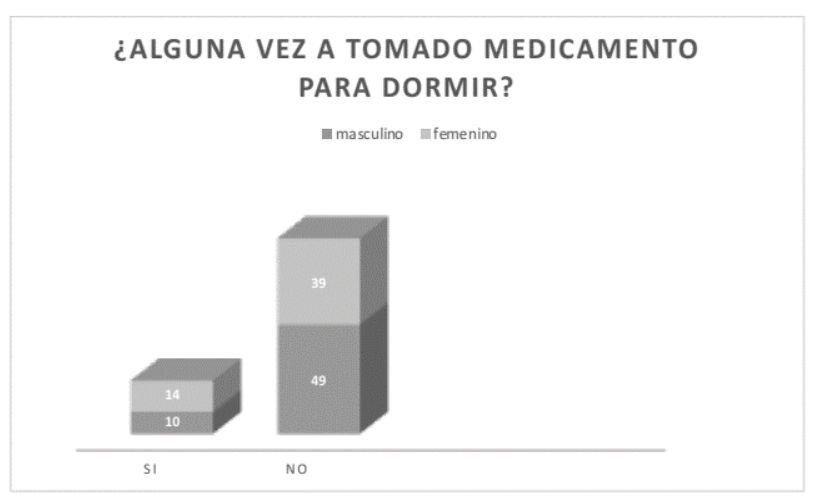

Grafico 5
Se puede apreciar que 24 de los jóvenes ha recurrido a algún medicamento para dormir y 88 afirman no tomar ningún medicamento para dormir.

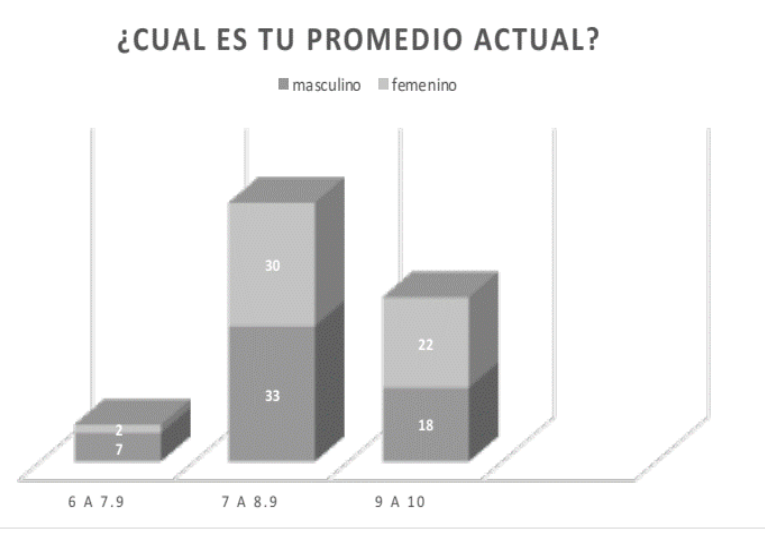

Grafico 6

Podemos observar que, la mayoría de los jóvenes (63) tiene un promedio regular de 7 a 8.9 , lo cual, puede tener diversos factores pero asociando las encuestas los que padecen algún tipo de trastorno o pocas horas de sueño afirman tener promedio de malo a regular, asi se puede observar la relación.

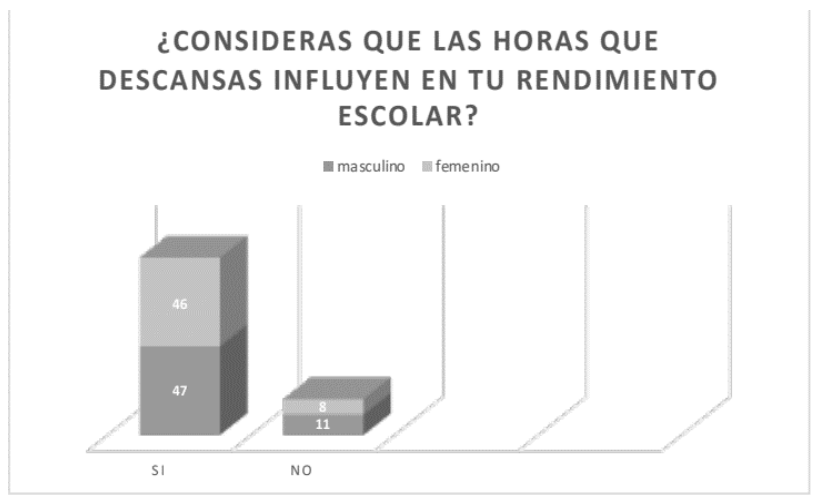

Grafico 7

Podemos observar que los jóvenes son conscientes de la importancia del sueño en su rendimiento académico, sin embargo, no cambian sus hábitos y planeación de tiempo para poder descansar las horas recomendadas medicamente para su etapa adolescente. 


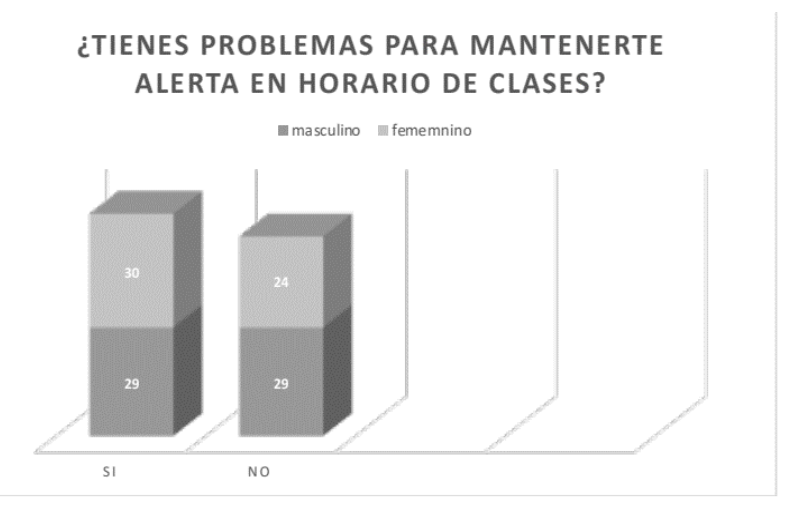

Grafico 8

Analizando la gráfica, se observa que la mayoría de los estudiantes encuestados tienen problemas o dificultad para mantenerse alerta en clases (59 jóvenes).

\section{Conclusiones}

Los alumnos del nivel Bachillerato muestran ciertos problemas para dormir, en los resultados se reflejó que los que están siendo más afectados son los varones ya que tienen un porcentaje más elevado que las mujeres, incluso se pudo analizar que sus horas de descanso no son las adecuadas, la mayoría de los alumnos duermen solo seis horas, y lo recomendado es que un adolescente duerma de nueve a diez horas, además de que los jóvenes suelen despertarse durante la noche e interrumpir su sueño, esto hace que su rendimiento académico pueda verse afectado , ya que la mayoría de los estudiantes tienen un promedio regular de 7 a 8.9 , por lo que se concluye que los jóvenes pueden llegar a subir su promedio escolar si duermen correctamente y así mismo prestar la suficiente atención para rendir en las diversas actividades escolares adecuadamente.

Un estudio del Instituto de neurociencia cognitiva de Londres informa que el cerebro continua desarrollándose después de la pubertad y que no está totalmente maduro hasta que superamos los 30 años, por lo que se recomienda tener un buena higiene de sueño y descanso, por ello, basándose en los resultados la Coordinación de Tutoría se propone diseñar curso o conferencia sobre la importancia de la planificación de tiempo y la importancia del sueño en el aprendizaje, esto para cumplir con su objetivo de apoyar al joven en sus dificultades académicas.

\section{Referencias}

Alva J.L. (2009) Consecuencias metabólicas y cardiovasculares del síndrome de apnea obstructiva del sueño. Med Int Mex. 25:116-28. García M., Salcedo F, Rodríguez F., Redondo M., Monterde M., Marcos A., Torrijos M. (2004) Prevalencia de los trastornos del sueño en adolescentes de Cuenca, España. Rev Neurol; 39:18-24.

García-Jiménez MA, Salcedo-Aguilar F, RodríguezAlmonacidb FM, et al: Prevalencia de los trastornos de sueño en adolescentes de Cuenca, España. Revista Neurology (Ed. Esp.) 2004; 39: 18-24.

Márquez J.M, Chiquete E. (2013) Frecuencia de insomnio y sus consecuencias diurnas en pacientes mexicanos: Subanálisis del estudio EQUINOX. Rev Mex Neuroci ;14:314-20.

Mental S, Sierra JC, Navarro CJ, Ortiz JDM (2002) Calidad del sueño en estudiantes universitarios: importancia de la higiene del sueño. Salud Mental; 25: 35.

P. Rubí G. (2018) Síndrome de piernas inquietas. Rev Med Chile 2018; 146: 1041-1049 